\title{
Volumetric comparative analysis of anatomy through far-lateral approach: surgical space and exposed tissues
}

Ke Tang ( $\nabla$ tkshoushudao@sina.com )

Feng $X u$

Xiaodong Yuan

Yang Li

Xinyue Chen

\section{Research}

Keywords: Far-lateral approach, Three-dimensional visualization, Surgical anatomy, Quantification, Minimally invasive

Posted Date: May 3rd, 2021

DOl: https://doi.org/10.21203/rs.3.rs-307279/v2

License: (9) (1) This work is licensed under a Creative Commons Attribution 4.0 International License.

Read Full License

Version of Record: A version of this preprint was published at Chinese Neurosurgical Journal on January 10th, 2022. See the published version at https://doi.org/10.1186/s41016-021-00268-8. 


\section{Abstract \\ Background}

The three-dimensional (3D) visualization model has ability to quantify the surgical anatomy of far-lateral approach. This study was designed to disclose the relationship between surgical space and exposed tissues in the far-lateral approach by the volumetric analysis of 3D model.

\section{Methods}

The 3D skull base models were constructed using MRI and CT data of 15 patients (30 sides) with trigeminal neuralgia. Surgical corridors of the far-lateral approach was simulated by triangular pyramids to represent two surgical spaces exposing bony and neurovascular tissues. Volumetric comparison of surgical anatomy was performed using pair $t$ test.

\section{Results}

The morphometric results were almost the same in the two surgical spaces except the vagus nerve (CN X) exposed only in one corridor. Whereas, the volumetric comparison represented the statistical significant differences of surgical space, and bony and neurovascular tissues involved in the two corridors (P®0.001). The differences of bony and neurovascular tissuesfailed to equal the difference of surgical space.

\section{Conclusions}

For far-laterl approach, the increase of exposure for the bony and neurovascular tissues is not necessarilymatched with the increase of surgical space. The volumetric comparative analysis is helpful to provide more detailed anatomical information in the surgical design.

\section{Background}

The lesions at the inferior clivus pose a surgical challenge with high morbidity and mortalityto neurosurgeons because ofthe critical neurovascular structures surrounding the lesion[1-4].Many studies have described the far-lateral approach which exposed theinferior clivus superior to the anterior edge of foramen magnum[5-8]. The concern of neurovascular protection and skull base reconstruction promotes the development of surgical anatomy for the far-lateral approach[9-11].

In the previous studies, The three dimensional (3D) visualization modelhad be utilized successfully in the assessment of surgical anatomy with advantage of providing quantitative details[12, 13]. The software of 3D model facilitate the volumetric measurement of anatomy[14, 15]. 
Researches had shown that the volumetric measurement was valuable toofferthe data for the design of minimally invasive surgery[16, 17]. It is an interesting issue in the surgical design to disclose the interrelation between the surgical space and exposed tissues. We thus sought to quantify the difference of volumes for the bony and neurovascular structures in the two surgical spaces of far-lateral approach.The relationship in the anatomical exposure generated by the surgical space was demonstrated in detail.

\section{Methods}

\section{Visualization of skull base}

Image data of MRI and CT was acquired during Gamma Knife surgery for 15 patients with trigeminal neuralgia. The 3D visualization model of skull base was constructed in a 3D software (Mimics,MaterialiseUS, Plymouth, Michigan). The 30 sides of skull base model were constructed for surgical simulation. The parameter of image acquisition and protocol for visualization of bony and neurovascular tissues has been reported previously[18].

\section{Surgical space for far-lateral approach}

The ipsilateral and contralateral anterior edge of jugular tuberculum, and anterior edge of foramen magnum were used as vertexes to form triangle representing exposed region. We thus named this triangle as exposed triangle. The center of exposed triangle was the intersection of three midlines. The posterior and superior edge of occipital condyle was used as landmark to draw a axis connect to the center of exposed triangle (Fig. 1).

The top of zygomatic process of frontal bone was selected as landmark to outline plane parallel to the exposed triangle.The intersection of the axis on the plane was the vertex to form triangular pyramid of the corridor 1 with exposed triangle as bottom. Similarly, the intersection between the axis andtemporal portion of calvarium was the vertex to form triangular pyramid of the corridor 2 (Fig. 1).

The line of triangular pyramid through contralateral anterior edge of jugular tuberculum crossed the occipital portion of calvarium to form a intersection which was used to outline craniotomy plane parallel to the exposed triangle. The triangular pyramid intersecting on the craniotomyplane to form craniotomy triangle. The space between the craniotomy and exposed triangle was define as surgical space for both corridor 1 and 2 (Fig. 1).

\section{Anatomical observation and volumetric analysis}

The sequence and morphology of bony and neurovascular structures in the surgical space were observed. The volume of exposed anatomical structures was calculated in the software. The volumetric comparison was performed bypair $t$ test of the SPSS software (SPSS16.0 for Windows; SPSS, Chicago, IL). The $p$ valueless than 0.05 was used to determine statistical significance. 


\section{Results}

\section{Exposure sequence}

The sequence of exposed anatomical tissues was almost the same in both corridor1 and 2 of far-lateral approach. The surgical space of simulative corridor started with bony drilling on the occipital condyle. The extra-cranial vertebral artery (VA) posterior to the occipital condyle was involved (Fig. 1).

The range of bony drilling in sequence involved the occipital condyle, occipitoatlantal joint, superior portion of lateral mass of atlas, the lateral edge of foramen magnum, posterior portion of jugular foramen and hypoglossal canal, inferior portion of jugular tuberculum and clivus above the foramen magnum. When bony drilling arrived at the jugular foramen, the surgical space contained posterior portion of glomus jugulare and the lower end of sigmoid sinus. The surgical space also involved the intra-canal portion of hypoglossal nerve (CN XII) when crossing hypoglossal canal.

For both corridor 1 and 2, the 16 sides of surgical space contained intra-cranial basilar artery (BA), whereas, 14 sides did not involved BA. The surgical space passed lateral and anterior to the cerebellum and medulla oblongata without brain tissues involved. The surgical space of corridor 1 and 2 contained the cisternal portion of accessory nerve (CN XI) and CN XII.Only surgical space of the corridor 2 involved CN X (Fig. 2).

\section{Volumetric analysis}

The volumetric comparison represented the significant differences of anatomical exposure between corridor 1 and 2 for far-lateral approach. The differences for all volumetric data reached statistical significance. The detail analysis was showed in table 1, of which the volume of surgical space in corridor 2 was almost twofold of that in corridor 1. However, instead of venous structures, the increase of bony and neurovascular structure exposed in corridor 2 than 1 failed to reach twofold. Especially for CN XII exposed, the increase in corridor 2 was not obvious.

\section{Discussion}

To date, 3D visualization model becomes more popular in the study of surgical anatomy for the merits of time-saving, reproducible, and manipulation without intra-operative pressure[19-21]. Based on the 3D visualization model containing bony and neurovascular tissues, the volumetric analysis can provide detailed information in the surgical anatomy in the skull base. We thus performed this anatomical study for the far-lateral approach using the 3D model.

\section{Value of simulative surgical corridor}

The pyramidal corridor simulating surgery can be understood as an illuminationrangelight source of operating microscope. The vertex of pyramid is the point directed by the light source. The bottom of pyramid represents the movement range of operating microscope. The 
surgical space in the pyramidal corridor therefore provides an intra-operative exposure range for anatomical structures. We designed two pyramidal corridors to simulated far-lateral approach exposing clivus, of which the surgical space in corridor 2 was approximate twofold than that in corridor 1.

The 3D simulative surgical corridor offers a critical condition for volumetric analysis. The result of morphometric comparative analysisonly showed that partial $\mathrm{CN} X$ was contained in the corridor 2 instead of corridor 1 . Whereas, the detail information of increased anatomical exposure in the corridor 2 was demonstrated objectively by the volumetric analysis.

\section{Volumetric analysis of bony tissues}

Regarding bony removal, the postoperative stabilization of the craniovertebral junctionis still a concern of life quality for the patient[22]. Stability rebuilding of craniovertebral junction was required for both corridor 1 and 2 following the intra-operative damage of occipitoatlantal joint. As the result of volumetric comparison showed, the more damage of occipitoatlantal joint occurred in corridor 2 which would face more difficulties in the stability reconstruction.

In addition, It was time-consuming procedure to perform bony drilling[23]. Obviously, the corridor 2 faced more difficulties in such manipulation for more volume of bony tissues involved. The more disturbance caused by the extra-cranial VA during bony drilling also increased the difficulty of corridor 2.

\section{Volumetric analysis of neurovascular tissues}

The surgical spaces of the two corridors exposed the cistern lateral and anterior to the cerebellum and medulla oblongata without disturbing the brain tissues, which may represent the condition of epidural manipulation to reach clivus in the far-lateral approach. When passing through the lateral edge of foramen magnum, the posterior portion of glomus jugulare and the inferior end of sigmoid sinus was involved, which has potential risk of troublesome bleeding[24, 25]. The increasing extent of venous structures in the surgical space of the corridor 2 was the most.

Compared with the approximate twofold increase of surgical space, the unmatched less increase of CN XI and XII was shown in the corridor 2, which implies that the difficulty of CN XI and XII protection was nearly similar in both corridor 1 and 2 . Meanwhile邓the $C N X$ was only involved in the corridor 2 . Careful manipulation should be performed to protect the cranial nerves[26].Due to the characteristics of position offset of the BA, only 16 sides of surgical surface involved partial BA of which the volume was slightly more in the corridor 2. The mobilization of BA can be used to improve the access.

\section{Technical limitations}

This anatomy study without real operative practice limit the clinical value. The regular geometric corridor simulating surgery is unable to complete in the current real operative conditions. We thus only demonstrate an objective comparison by volumetric analysis via regular corridors. This article highlights 
the relevant valuesof quantification forthe surgical space and anatomical exposure in reference to the technical development of 3D visualization and neuronavigation.

\section{Conclusions}

We found that the increase of surgical space exposing clivus via simulative far-lateral approach was majorly used to increase the volume of bony and venous structures. The surgical space in the corridor 1 was more minimally invasive than thecorridor 2 . The detailed information can be provided by the volumetric comparison.

\section{Abbreviations}

3D:three dimensional; BA: basilar artery; CN X: vagus nerve; CNXI : accessory nerve; CN XII: hypoglossal nerve; GJ: glomus jugulare; MO: medulla oblongata; SS:sigmoid sinus; VA: vertebral artery.

\section{Declarations}

\section{Funding}

Capital health development research special fund(2014-4-5073)

Educational science and technology projects for young and middle-aged teachers of Fujian Provincial Department of Education囚JAY190824】

\section{Authors' contributions}

Ke Tang and Xu Feng designed the study; Xu Feng and Xinyue Chen constructed the 3D skull base models ;Xiao dong Yuan and Yang Li collected and analyzed the data; Ke Tang and Xu Feng analyzed the data and wrote the manuscript; Ke Tang, Xu Feng, Xiaodong Yuan, Yang Li and Xinyue Chen performed the experiments; and all the authors have read the manuscript and approved its submission.

\section{Availability of data and materials}

The datasets generated during and analyzed during the current study are available from the corresponding author on reasonable request.

\section{Ethics approval and consent to participate}

The current study was approved by the Ethics Committee of The First Medical Center of Chinese PLA General Hospital.

Written informed consent was obtained from all participants. 


\section{Consent for publication}

Not applicable.

\section{Competing interests}

The authors declare no potential conflict of interest.

\section{Author details}

${ }^{1}$ Institute of Neurosurgery, The First Medical Center of Chinese PLA General Hospital, Fuxing Road 28, Beijing 100853, P.R. China; ${ }^{2}$ Department of Basic Medicine, Xiamen Medical College, Guan kou zhong Road 1999, Xiamen, Fujian Province, 361023,P.R. China; ${ }^{3}$ Department of Neurosurgery, The Eighth Medical Center of Chinese PLA General Hospital, Heishanhu Road 17, Beijing, 100091, P.R. China;

${ }^{4}$ Departmentof Oral and Maxillofacial Surgery, Peking University School and Hospital of Stomatology, Zhong guan cun South Road 22, Beijing 100081, P.R. China;

\section{References}

1. Beer-Furlan A, Balsalobre L, Vellutini EA, Stamm AC. Endoscopic endonasal approach in invasive aspergillosis of the clivus in an immunocompetent patient. Acta Neurochir (Wien). 2015 Dec;157(12):2221-2.

2. Cheng Y, Zhang S, Chen Y, Zhao G. Safe Corridor to Access Clivus for Endoscopic Trans-Sphenoidal Surgery: A Radiological and Anatomical Study. PLoS One. 2015;10(9):e0137962.

3. Scerrati A, Ercan S, Wu P, Zhang J, Ammirati M. Intrapetrous Internal Carotid Artery: Evaluation of Exposure, Mobilization and Surgical Maneuvers Feasibility from a Retrosigmoid Approach in a Cadaveric Model. World Neurosurg. 2016 Jul;91:443-50.

4. Kshettry VR, Healy AT, Colbrunn R, Beckler DT, Benzel EC, Recinos PF. Biomechanical evaluation of the craniovertebral junction after unilateral joint-sparing condylectomy: implications for the far lateral approach revisited. J Neurosurg. 2017 Oct;127(4):829-36.

5. Liu J, Ahmadpour A, Bewley AF, Lechpammer M, Bobinski M, Shahlaie K. Chondroblastoma of the Clivus: Case Report and Review. J Neurol Surg Rep. 2015 Nov;76(2):e258-64.

6. Shiban E, Torok E, Wostrack M, Meyer B, Lehmberg J. The far-lateral approach: destruction of the condyle does not necessarily result in clinically evident craniovertebral junction instability. J Neurosurg. 2016 Jul;125(1):196-201.

7. La Corte E, Aldana PR. Endoscopic approach to the upper cervical spine and clivus: an anatomical study of the upper limits of the transoral corridor. Acta Neurochir (Wien). 2017 Apr;159(4):633-39.

8. Singh H, Patir R, Vaishya S, Gupta A, Miglani R. Application of a Far-Lateral Approach to the Subaxial Spine: Application, Technical Difficulties, and Results. World Neurosurg. 2017 Apr;100:167-72. 
9. Liebelt BD, Boghani Z, Haider AS, Takashima M. Endoscopic repair technique for traumatic penetrating injuries of the clivus. J Clin Neurosci. 2016 Jun;28:152-6.

10. Wang K, Wang L, Wu Z, Tian K, Ren C, Jia G, et al. Bone invasiveness is associated with prognosis in clivus chordomas. J Clin Neurosci. 2016 May;27:147-52.

11. Fukuda H, Evins Al, Iwasaki K, Hattori I, Murao K, Kurosaki Y, et al. The role of alternative anastomosis sites in occipital artery-posterior inferior cerebellar artery bypass in the absence of the caudal loop using the far-lateral approach. J Neurosurg. 2017 Feb;126(2):634-44.

12. Besharati Tabrizi L, Mahvash M. Augmented reality-guided neurosurgery: accuracy and intraoperative application of an image projection technique. J Neurosurg. 2015 Jul;123(1):206-11.

13. Qian ZH, Feng X, Li Y, Tang K. Virtual Reality Model of the Three-Dimensional Anatomy of the Cavernous Sinus Based on a Cadaveric Image and Dissection. J Craniofac Surg. 2017 Oct 18.

14. Ors S, Inci E, Turkay R, Kokurcan A, Hocaoglu E. Retrospective comparison of three-dimensional imaging sequences in the visualization of posterior fossa cranial nerves. Eur J Radiol. 2017 Dec;97:65-70.

15. Qian Z, Li Y, Feng X, Li Y, Tang K. Quantification of Microsurgical Anatomy in Three-Dimensional Model: Transfrontal Approach for Anterior Portion of the Thalamus. J Craniofac Surg. 2019 May/Jun;30(3):926-29.

16. Adams Perez J, Rassier Isolan G, Pires de Aguiar PH, Antunes AM. Volumetry and analysis of anatomical variants of the anterior portion of the petrous apex outlined by the kawase triangle using computed tomography. J Neurol Surg B Skull Base. 2014 Jun;75(3):147-51.

17. Freeman JL, Sampath R, Quattlebaum SC, Casey MA, Folzenlogen ZA, Ramakrishnan VR, et al. Expanding the endoscopic transpterygoid corridor to the petroclival region: anatomical study and volumetric comparative analysis. J Neurosurg. 2017 Jul 21:1-10.

18. Tang K, Qian ZH, Feng X, Li Y. Gridding Microsurgical Anatomy of Far Lateral Approach in the ThreeDimensional Model. J Craniofac Surg. 2019 Jan;30(1):87-90.

19. Kanowski M, Voges J, Buentjen L, Stadler J, Heinze HJ, Tempelmann C. Direct visualization of anatomic subfields within the superior aspect of the human lateral thalamus by MRI at 7T. AJNR Am J Neuroradiol. 2014 Sep;35(9):1721-7.

20. Winkler-Schwartz A, Bajunaid K, Mullah MA, Marwa I, Alotaibi FE, Fares J, et al. Bimanual Psychomotor Performance in Neurosurgical Resident Applicants Assessed Using NeuroTouch, a Virtual Reality Simulator. J Surg Educ. 2016 Nov - Dec;73(6):942-53.

21. Pelargos PE, Nagasawa DT, Lagman C, Tenn S, Demos JV, Lee SJ, et al. Utilizing virtual and augmented reality for educational and clinical enhancements in neurosurgery. J Clin Neurosci. 2017 Jan;35:1-4.

22. Ji W, Tong J, Huang Z, Zheng M, Wu X, Chen J, et al. Stabilization of the Craniovertebral Junction with Clivus Plate Constructs: Biomechanical Comparison with Conventional Technique. World Neurosurg. 2016 Oct;94:42-49. 
23. Xiao X, Wu Z, Zhang L, Jia G, Tang J, Meng G, et al. Using a modified far-lateral approach to remove hypoglossal neurilemmomas: notes on technique. J Neurosurg. 2013 Mar;118(3):657-68.

24. Patel SK, Liu JK. Staged bilateral far-lateral approach for bilateral cervicomedullary junction neurenteric cysts in a 10-year-old girl. J Neurosurg Pediatr. 2013 Sep;12(3):274-80.

25. Mizutani K, Toda M, Yoshida K. The Analysis of the Petrosal Vein to Prevent Venous Complications During the Anterior Transpetrosal Approach in the Resection of Petroclival Meningioma. World Neurosurg. 2016 Sep;93:175-82.

26. Salle H, Cavalcanti Mendes GA, Gantois C, Lerat J, Aldahak N, Caire F. Endoscopic Submandibular Retropharyngeal Approach to the Craniocervical Junction and Clivus: an Anatomic Study. World Neurosurg. 2017 Oct;106:266-76.

\section{Table}

TABLE 1. Volumetric comparison of surgical anatomy

\begin{tabular}{llllll}
\hline Item & \multicolumn{2}{l}{ Corridor $1\left(\mathrm{~mm}^{3}\right)$} & \multicolumn{2}{l}{ Corridor $2\left(\mathrm{~mm}^{3}\right)$} & $\mathrm{p}$ Value \\
& Mean & $\mathrm{SD}$ & Mean & $\mathrm{SD}$ & \\
\hline Operative space & 5501.60 & 150.03 & 11430.00 & 1085.88 & $\square 0.001$ \\
\hline Bony drilling & 1745.50 & 112.54 & 2763.50 & 145.15 & $\square 0.001$ \\
\hline Extra-cranial VA & 64.05 & 1.34 & 95.53 & 1.08 & $\square 0.001$ \\
\hline BA & 28.90 & 1.84 & 37.86 & 1.41 & $\square 0.001$ \\
\hline Venous structures & 72.36 & 1.63 & 205.69 & 14.10 & $\square 0.001$ \\
\hline CN X † & - & - & 5.50 & 0.20 & - \\
\hline CN XI & 9.61 & 0.40 & 15.38 & 1.21 & $\square 0.001$ \\
\hline CN XII & 11.20 & 1.07 & 13.55 & 1.23 & $\square 0.001$ \\
\hline
\end{tabular}

Comparison of the volumes $\left(\mathrm{mm}^{3}\right)$ measured in corridor 1 and 2 (30 sides). p values $<0.05$ indicate statistical significance. $\mathrm{BA}=$ basilar artery; $\mathrm{CN} \mathrm{X}$ = vagus nerve; $\mathrm{CN} \mathrm{XI}=$ accessory nerve; $\mathrm{CN} \mathrm{XII} \mathrm{=}$ hypoglossal nerve; $\mathrm{VA}=$ vertebral artery.

* BA was involved in 16 sides of 3D model.

† Only the corridor 2 involved CN X. Thus, the Pair t test was not performed.

\section{Figures}



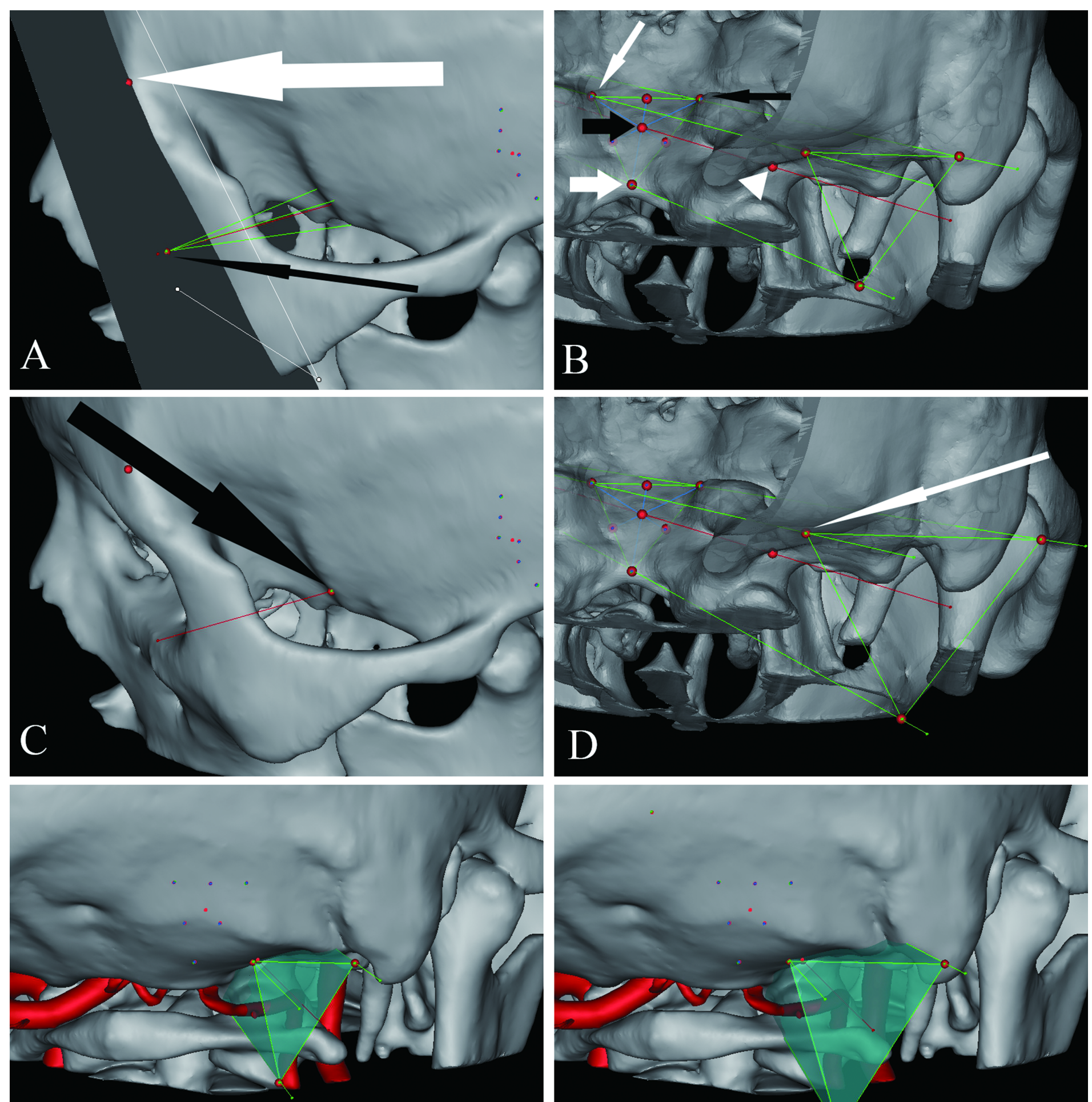

$\mathrm{E}$

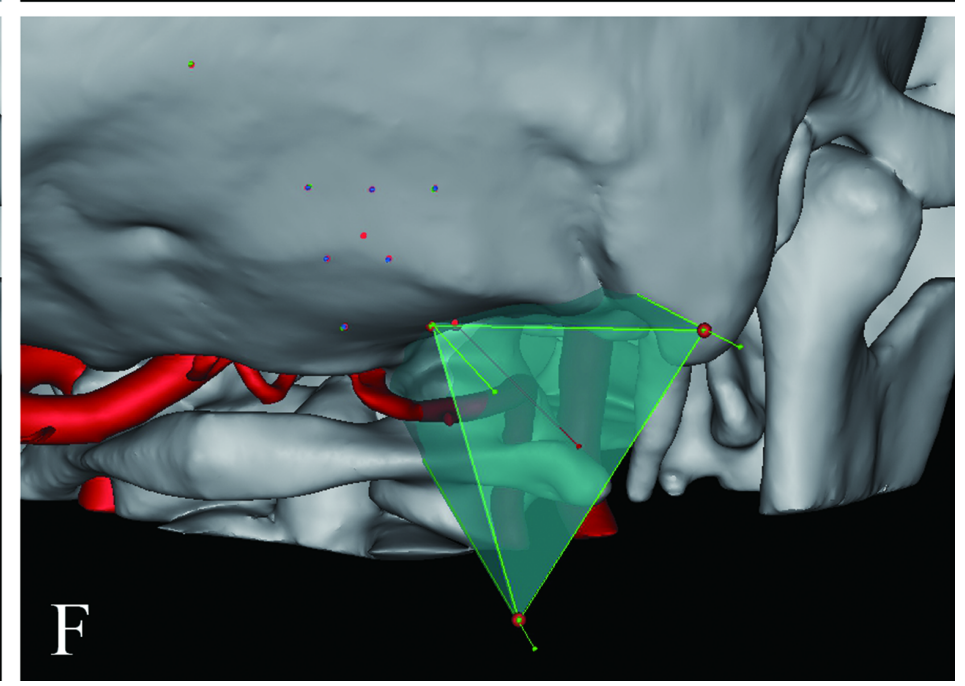

Figure 1

Surgical corridors outlined based on bony landmarks. A: The top of zygomatic process of frontal bone (white thick long arrow) was used to outline plane parallel to the exposed triangle. The intersection (black thin long arrow) of the plane and the axis was the vertex of triangular pyramid for corridor 1. B: The exposed triangle was outlined with the ipsilateral (black thin short arrow) and contralateral (white thin short arrow) anterior edge of jugular tuberculum, and anterior edge of foramen magnum (white thick 
short arrow) as vertexes. The axis was drawn to connect the center (black thick short arrow) of exposed triangle and the posterior and superior edge of occipital condyle (white arrow head). C: The intersection (black thick long arrow) between axis and calvarium was the vertex of triangular pyramid for corridor 2. D: The line on the triangular pyramid through contralateral anterior edge of jugular tuberculum form intersection (white thin long arrow) on the calvarium. Posterior view of the corridor 1 (E) and corridor 2 $(\mathrm{F})$.
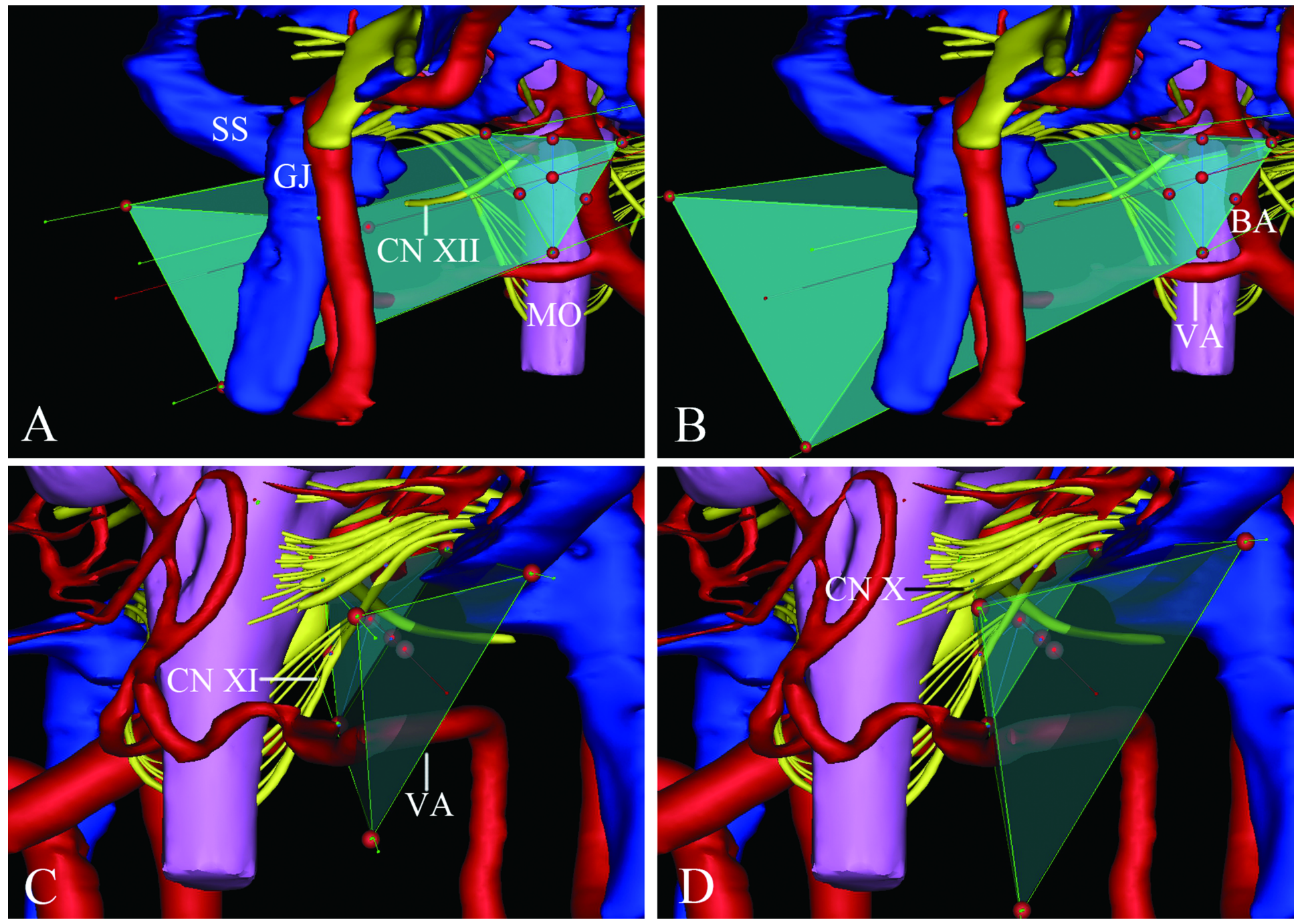

Figure 2

Relationship between the surgical space and neurovascular structures. A and B: Anterior view of 3D reconstruction of neurovascular structures and corridor 1 and 2. $C$ and D: Posterior view of 3D reconstruction of neurovascular structures and corridor 1 and 2. $\mathrm{BA}=$ basilar artery; $\mathrm{CN} X=$ vagus nerve; $\mathrm{CN} \mathrm{XI}=$ accessory nerve; $\mathrm{CN} \mathrm{XII} \mathrm{=hypoglossal} \mathrm{nerve;} \mathrm{GJ}$ = glomus jugulare; $\mathrm{MO}$ = medulla oblongata; $\mathrm{SS}$ =sigmoid sinus; $\mathrm{VA}=$ vertebral artery. 